\title{
A integração do ensino de funções trigonométricas e movimento harmônico simples por meio do software Modellus
}

\author{
The integration of the teaching of trigonometric functions and simple harmonic motion through Modellus
} software

\author{
Claudionor de Oliveira Pastana*1, Italo Gabriel Neide ${ }^{2}$ \\ ${ }^{1}$ Universidade do Estado do Amapá, Colegiado de Engenharia Florestal, Presidente Vargas, 650, Macapá, AP, Brazil \\ ${ }^{2}$ Centro Universitário Univates, Lajeado, RS, Brazil
}

Recebido em 23 de Março, 2017. Revisado em 28 de Maio, 2017. Aceito em 07 de Julho, 2017.

\begin{abstract}
Este trabalho aborda o ensino de funções trigonométricas associado ao Movimento Harmônico simples por meio do software Modellus. O trabalho é parte de uma pesquisa de Mestrado Profissional em Ensino de Ciências Exatas, do Centro Universitário UNIVATES. O estudo foi realizado em uma escola pública da rede estadual do município de Macapá, Amapá, tendo, como participantes, trinta e seis estudantes do $3^{\circ}$ ano do Ensino Médio. O objetivo desta pesquisa é investigar as implicações de utilizar o Software Modellus, para ensinar os conceitos de Funções Trigonométricas por meio do Movimento Harmônico Simples, em uma turma do $3^{\circ}$ ano do Ensino Médio da Educação Básica na cidade de Macapá - AP. A pesquisa desenvolvida é de natureza qualitativa abordando um estudo de caso. Para análise e coleta dos dados, foram feitas observações, aplicação de um questionário prévio e desenvolvimento de atividades pedagógicas com o uso do software Modellus. Os resultados da pesquisa indicaram que as atividades pedagógica desenvolvida com os alunos mostrou ser potencialmente relevante, pois apresentou indícios de novos conceitos de funções trigonométricas integrados ao movimento harmônico simples, auxiliou no enriquecimento, modificação e elaboração.
\end{abstract}

Palavras-chave: Ensino de Matemática e Física, recursos tecnológicos, software Modellus.

This work deals with the teaching of trigonometric functions associated with the Simple Harmonic Motion through the Modellus software. The work is part of a research of a Master's Degree in Teaching of Exact Sciences, of the University Center UNIVATES. The study was made in a public state school of Macapá city, in Amapá, having as participants, thirty-six students in the $3^{\text {rd }}$ High year. The purpose of this research is to investigate the implications of using the Modellus Software to teach the concepts of Trigonometric Functions through the Simple Harmonic Movement in a class of the $3^{\text {rd }}$ high school year of Basic Education in Macapá - AP. The developed research has a qualitative nature, approaching a case study. For the analysis and data collection, observations, application of a previous questionnaire and development of pedagogical activities were done using the Modellus software. The results of the research indicated that the pedagogical activities developed with the students showed to be potentially relevant, as they presented indications of new concepts of trigonometric functions integrated to the simple harmonic movement, helped in the enrichment, modification and elaboration.

Keywords: Mathematics and Physics teaching, technological resources, Modellus software.

\section{Introdução}

A exploração de recursos tecnológicos, em particular do computador, está possibilitando consideráveis avanços nos processos de ensino de Matemática e de Física em diversos estabelecimentos educacionais [1]. Nesse sentido, o emprego dos recursos tecnológicos em sala de aula pressupõe, obrigatoriamente, uma reflexão sobre a prática pedagógica desenvolvida pelos docentes, no sentido de contribuir com os processos de ensino e de aprendizagem. Vale ressaltar, porém, que muitas vezes a utilização desses recursos não significa avanço na prática pedagógica [2].

*Endereço de correspondência: claudionorpastana@yahoo.com.br
No ambiente escolar existem algumas experiências em relação ao uso dos recursos computacionais em situações de aprendizagem no ensino de Matemática e Física, assim como nas outras disciplinas de Ciências da Natureza e suas Tecnologias. Essas práticas educacionais, porém, poderiam ser mais presentes e interligadas às diversas áreas de conhecimento [3].

O processo de interligação entre as diversas disciplinas do currículo escolar poderia ser mais bem articulado. Para fazê-lo, há diversas formas, mas uma, em potencial, poderia ser a utilização de tecnologias, em particular no desenvolvimento de modelagem de fenômenos naturais em softwares educativos. Para [4] softwares educacionais 
se diferem de outros recursos, pelo fato de proporcionarem um feedback imediato ao educando e educador, possibilitando a reorganização das práxis educativas.. Esses softwares fazem uso de modelagem computacional, que permite a demonstração de fenômenos naturais, aproximando a realidade vivenciada pelo discente com o processo de ensino e de aprendizagem.

\section{Fundamentação}

As discussões desenvolvidas e propostas que faremos nesse trabalho são baseadas especialmente nas Diretrizes Curriculares Nacional do Ensino Médio - DCNEM. As Diretrizes Curriculares Nacionais do Ensino Médio - DCNEM, preconizam uma prática escolar mais integral, moderna e sua execução pautada no "[...] trabalho como princípio educativo e a pesquisa como princípio pedagógico", [5].

Por estes motivos, [5] ainda destaca que torna-se imprescindível organizar as disciplinas, os espaços e os tempos escolares para obter um ensino que instigue os educandos no desenvolvimento de sua aprendizagem. Simultaneamente promovendo "a integração dos conhecimentos de todas as áreas de conhecimento, articuladas pelas dimensões do trabalho, cultura, ciência e tecnologia" [5].

Naturalmente, existe mais uma provocação para os docentes na elaboração de atividades que "contemplem de maneira efetiva a construção de conhecimentos de seu componente curricular, integrada a outros componentes e/ou áreas" [5]. Essa integração entre os diferentes componentes curriculares, surge a partir de características e conhecimentos próprios dessas áreas, permitindo propor atividades que estimulem a participação dos discentes, citado pela mesma obra. Além disso, esta integração também deve acontecer sob o aspecto do contexto em que vive o discente, ocorrendo uma ligação entre disciplinas, realidade, conhecimento, discentes e docentes buscando ensinar e aprender.

Dessa forma, os docentes das áreas de Matemática e Física necessitam reconsiderar e reconhecer as possibilidades de colaboração em atividades integradoras, baseadas nos conhecimentos que são próprios, articulados a contextos autênticos e que sejam consideráveis para a formação integral dos educandos [5]. De acordo com a obra, é considerável verificar a abrangência da conexão da Matemática com a Física em diferentes contextos sociais e culturais.

No processo de integração da Matemática com a Física, a escolha de um tema que facilite essa conexão, não essencialmente está conectada "[...] com uma simples curiosidade sobre o funcionamento do mundo, mas envolve também influências sociais, culturais, políticas e econômicas" [6]. Há diversas formas de integrar e conectar os processos de ensino e de aprendizagem em favor do discente, assim a influência da realidade em que ele está inserido deve ser a base para a construção do vínculo entre as disciplinas e entre elas e os discentes.
Assim, exige-se cada vez mais dos docentes uma postura de saber utilizar as novas ferramentas tecnológicas de apoio, tanto laboral como socialmente ao alcance do objetivo na educação: a formação integral do educando [7]. Para [1] "as dimensões da inovação tecnológica permitem a exploração e o surgimento de cenários alternativos para a educação [...]". Esse cenário tecnológico proporciona ao professor e ao aluno um dinamismo na interação com o assunto facilitando a aprendizagem do tema estudado.

Além disso, o processo de inovação no meio tecnológico está cada vez mais eficiente e útil, extremamente ágil, exigindo uma adaptação constante de seus usuários. "A forma acelerada com que inovações tecnológicas vêm tomando corpo é, atualmente, uma característica marcante de nossa sociedade [...]", [1]. No entendimento dos autores, essas inovações poderão contribuir com o fenômeno educativo por meio de estímulos, vivências e provocações estabelecidas por estratégias de ensino. [8] destaca que:

Há uma disseminação geral das tecnologias da informação e comunicação; elas estão presentes e influenciam a vida social. Neste sentido não se pode negar o relacionamento entre o conhecimento no campo da informática e os demais campos do saber humano. Trata-se de uma nova forma de linguagem e de comunicação, um novo código: a linguagem digital $[\ldots]$.

De acordo com [9] os softwares de modelagem são ferramentas computacionais que facilitam a construção de modelos matemáticos que representam o cotidiano, tornando os processos de ensino e de aprendizagem mais próximo da realidade do aluno. Além disso, "dentre as ferramentas de modelagem disponíveis atualmente, o Software Modellus destaca-se por permitir que estudantes e professores façam experimentos conceituais utilizando modelos matemáticos [...]".

O Software Modellus é um programa computacional de distribuição gratuita por meio da internet, sua criação é obra da Faculdade de Ciências e Tecnologias da Universidade Nova de Lisboa. [10] destaca que o software Modellus foi desenvolvido, e encontra-se constantemente sendo aperfeiçoado, por um grupo de estudiosos da Faculdade de Ciências e Tecnologia da Universidade Nova de Lisboa, liderados pelo Professor Vitor Duarte Teodoro. É um software que simula e constrói modelos de fenômenos Químicos, Matemáticos e Físicos "[...] o qual permite ao aluno realizar e construir experimentos conceituais utilizando modelos matemáticos definidos a partir de funções [...]", [11]. Além disso,

O Software Modellus permite que alunos e professores realizem experiências proporcionadas pela construção e manipulação de modelos matemáticos para a resolução de cálculos e construção de gráficos que permitem uma exploração mais dinâmica e interativa [...], [12]. 
Entre as muitas possibilidades de uso de uma ferramenta computacional, para modelar situações e fenômenos do mundo real, no contexto do ensino, o Software Modellus é uma das mais poderosas, visto que, sua distribuição é gratuita e não é necessário estar conectado à internet [13]. Já "do ponto de vista computacional, o programa pode ser visto como um micromundo no computador para uso tanto dos estudantes quanto dos professores, não sendo baseado numa metáfora de programação [...]" $[9]$.

\section{Procedimento Metodológico}

A natureza do desenvolvimento dessa pesquisa foi de cunho qualitativo. A pesquisa "qualitativa engloba a ideia do sujeito, passível de expor sensações e opiniões [...]", [14]. A pesquisa qualitativa possibilita autonomia para a pessoa pensar e se expressar em relação ao assunto exposto. Em vez de um resultado definitivo, há diversas interpretações, levando em consideração as opiniões, os argumentos e comentários dos sujeitos da pesquisa.

Os referidos autores ressaltam que os estudos de pesquisa qualitativa diferem entre si quanto ao método, à forma e aos objetivos de abordagem. Neste tipo de pesquisa considera-se que há uma relação dinâmica entre o sujeito e o mundo real, e o conhecimento não se constrói baseando-se apenas em dados isolados, analisados de maneira dissociável.

Como a proposta de desenvolvimento dessa pesquisa envolveu uma investigação e intervenção de âmbito pedagógico em um contexto autêntico de sala de aula, compreendo que essa abordagem seja de cunho qualitativo. Para [14], "[...] é o caminho para escapar da mesmice. Lida e dá atenção às pessoas e as suas ideias, procurando fazer sentido de discursos e narrativas que estariam silenciosas [...]". Um dos enfoques da pesquisa qualitativa são as pessoas e suas atividades, considerando suas interpretações à medida que interagem com outros e refletem a respeito de suas experiências e atividades cotidianas.

Participaram desta pesquisa os 36 alunos da turma, composta de 23 estudantes do sexo feminino e 13 do sexo masculino. As atividades de pesquisa foram realizadas no turno e horário das aulas de Matemática, durante quatro semanas, tendo dois encontros semanais, um de duas horas aula de cinquenta minutos e outro de uma hora aula de cinquenta minutos cada. A pesquisa foi realizada em uma Escola Pública Estadual da Cidade de Macapá, AP que concedeu o termo de concordância para realização da pesquisa.

Buscando a melhor transcrição e compreensão das respostas apresentadas pelos estudantes, optou-se por elencar algumas questões norteadoras. Para o desenvolvimento da pesquisa foi realizado a aplicação de um questionário estruturado prévio e a realização de atividades pedagógicas, abordando Funções Trigonométricas integradas ao Movimento Harmônico Simples, por meio do Software Modellus.

\section{Análise dos Resultados}

Para a melhor compreensão do leitor, a análise dos resultados foi dividida em duas subseções. A primeira apresenta a análise qualitativa de uma atividade do questionário estruturado prévio e a segunda, a análise de uma atividade pedagógica com o Software Modellus.

\subsection{Análise do questionário estruturado prévio}

Na sequência, a Figura 1 apresenta a quantidade de respostas que convergiram para os conceitos de funções trigonométricas e movimento harmônico simples e as que divergiram desses conceitos, repassada pelos estudantes no questionário estruturado prévio.

Como podemos perceber pela figura 1 na pergunta do questionário prévio a maioria dos alunos realizaram cálculos incompreensíveis ou operações erradas, seguido de respostas não conexas aos conceitos de Funções trigonométricas ou de Movimento Harmônico simples. Poucos alunos possuíam realizaram respostas conexas aos conceitos Matemáticos e Físicos esperados.

A primeira pergunta do questionário estruturado prévio possuía três indagações dissertativas e conexas ao comportamento da função seno, relacionando com o comportamento do movimento harmônico simples: "O sistema cardiovascular do organismo humano, do qual o coração é o órgão principal, tem a finalidade de fazer circular o sangue pelo corpo. A quantidade de contrações realizadas por minuto determina a intensidade de frequência cardíaca, que oscila de acordo com as necessidades do organismo. Um atleta, por exemplo, pode ter a frequência cardíaca descrita pela função: $h(t)=2+3$.sin $\left(\frac{\pi \cdot t}{36}\right)$, em que t é o tempo medido em segundos". Vale ressalta que a função $h(t)=2+3 \cdot \sin \left(\frac{\pi \cdot t}{36}\right)$, representa um modelo matemático simplificado dos aspectos reais da frequência cardíaca.

Pudemos verificar que os quatro alunos responderam à pergunta com uma conexão a respeito da característica da função, porém, realizaram cálculos incompreensíveis. Observando as respostas dos alunos e os comentários

\section{Análise do questionário prévio}

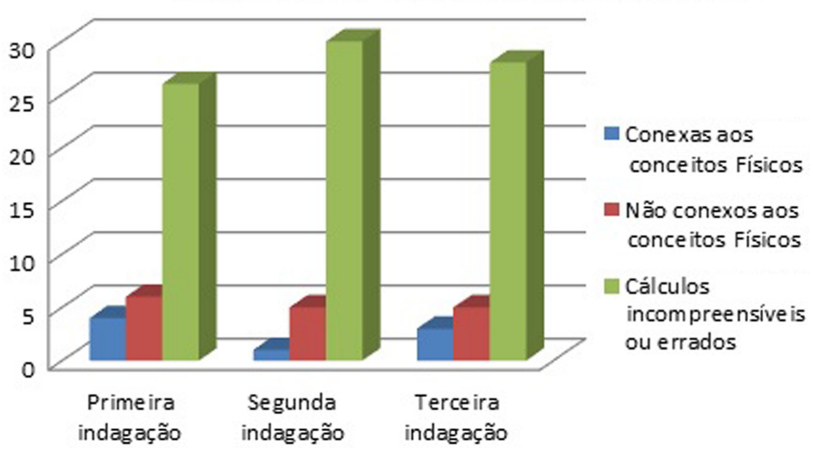

Figura 1: Gráfico das respostas do questionário estruturado prévio, dos autores (2017). 
nas transcrições, observamos que os alunos seis alunos participantes da pesquisa responderam sem nexo, como se não tivessem entendido a pergunta ou não possuíssem conhecimento a respeito de Funções Trigonométricas, como mostra a Figura 2:

Por meio das respostas dos seis alunos, foi possível perceber que os mesmo não sabiam quais as características de uma função trigonométrica aplicada em outras áreas do conhecimento, uma relação que apresenta indícios que os alunos não estão habituados a realizar. Observamos também, de imediato, que os alunos procuravam realizar cálculos sem tentar compreender o que o problema solicitava.

Nessa pergunta, pudemos considerar correta uma resposta, de acordo com as concepções físicas atualmente aceitas: o aluno descreveu a periodicidade do movimento em um intervalo de tempo. Para [15], uma das características do gráfico de uma função periódica de período t é que o mesmo se repete em cada intervalo do comprimento do período.

Além dos parâmetros da função seno, também buscamos compreender o entendimento dos alunos sobre as funções trigonométricas, que [15] enfatiza serem modelações matemáticas de fenômenos periódicos. Na segunda indagação da primeira pergunta, percebemos que cinco alunos não souberam relacionar período e amplitude de uma função trigonométrica no movimento harmônico simples. Os alunos realizaram a soma dos parâmetros da função, dando respostas como a da Figura 3:

A função $h(t)=2+3$. $\sin \left(\frac{\pi \cdot t}{36}\right)$ representava o sistema harmônico cardiovascular do ser humano, responsável por fazer a circulação do sangue no corpo. Observando a Figura 2, o aluno somou o valor do parâmetro A com do parâmetro B. Uma das grandezas relacionada à frequência

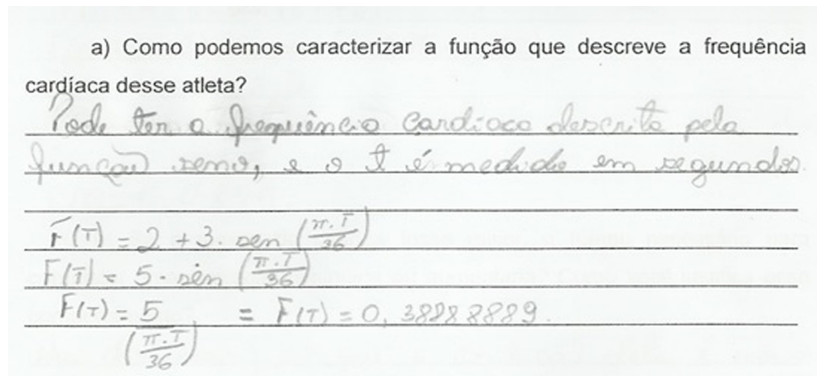

Figura 2: Resposta de um aluno que realizou cálculos errados, dos autores (2017).

b) Qual seria o periodo e amplitude entre um batimento e outro? Operiodo de batimente e 5 segundo 5 por -5
Datimento.

Figura 3: Resposta de um aluno que realizou cálculos errados, dos autores (2017). é o período T de oscilação do movimento que constitui o tempo necessário para completar um ciclo de oscilação do movimento harmônico simples e a frequência, é o número de oscilações completas por segundo [16].

Verificando as respostas dos demais alunos participantes da pesquisa, constatamos que nenhum acertou qual seria a amplitude e o período do movimento. A amplitude variava de [-1 até 5] e o período era de 72 segundos.

Observando a resposta dos alunos com relação à terceira arguição da primeira pergunta do questionário estruturado prévio, constatei que nenhum dos alunos conseguiu realizar o esboço da função em estudo. Cinco alunos, confundiram os elementos e características da função seno com a função cosseno. Vale destacar que, observando as respostas dos alunos e as transcrições das conversas, para os alunos as funções trigonométricas possuíam o mesmo gráfico, sem distinção ou características diferentes. Muitos apresentaram respostas como a apresentada na Figura 4:

No esboço da figura 4, é possível perceber que os alunos não descreveram as características do gráfico da função seno e não atribuíram variáveis as dimensões do sistema de coordenadas cartesianas. [17] destaca que:

\section{O gráfico da função periódica se caracteriza por apresentar um elemento de curva que se repete, isto é, se quisermos desenhar toda a curva bastará construir um caminho onde está desenhando o tal elemento de curva e ir carimbando. Período é o comprimento do carimbo (medido no eixo dos $\mathrm{x}$ ).}

Percebemos que os alunos não conseguiram esboçar o gráfico da função, destacando suas características. Uma resposta matemática aceitável seria um esboço em que o gráfico passasse pela origem dos eixos de coordenadas cartesianas e sua imagem fosse [-1;5]. Apenas um aluno apresentou um gráfico semelhante a uma função senoidal; os demais alunos traçaram esboço de funções afim, quadráticas e de circunferências.

\subsection{Análise das atividades com o Software Modellus}

A atividade de integração de funções trigonométricas com o movimento harmônico simples possuía questões

c) Esboce e descreva as principais caracteristicas do gráfico da função que representa o sistema cardiovascular desse atleta.

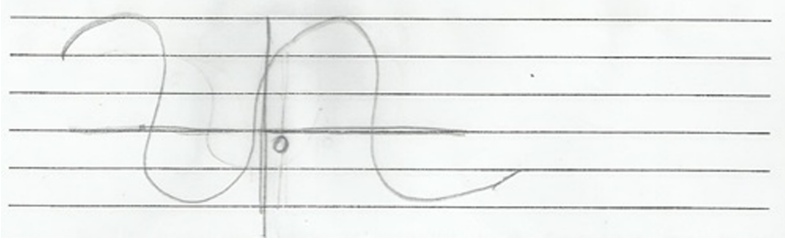

Figura 4: Resposta de um aluno que não soube interpretar, dos autores (2017). 


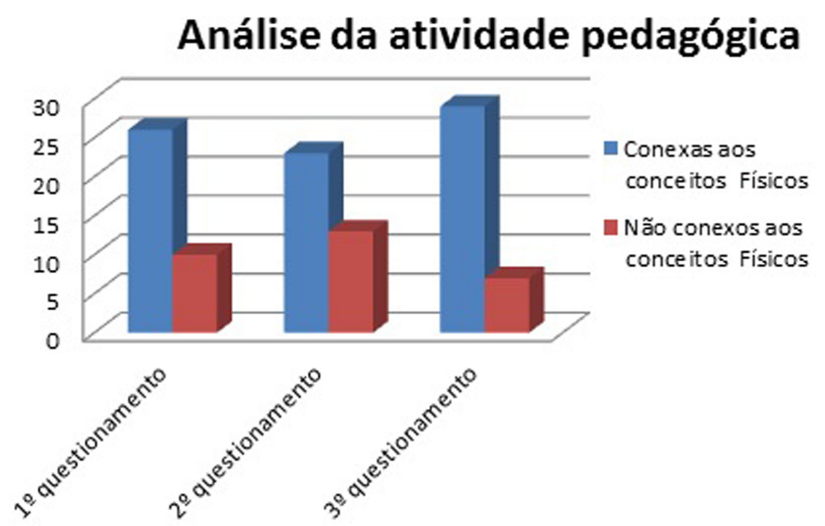

Figura 5: Gráfico da atividade com o Software Modellus, dos autores (2017).

dissertativas. Buscamos, com essa atividade, possibilitar a construção de conceitos de função trigonométrica, integrados aos do movimento harmônico simples por meio do Software Modellus. Na sequência, a Figura 5 apresenta a quantidade de respostas que convergiram de forma correta para os conceitos de funções trigonométricas e movimento harmônico simples por meio das atividades com o software Modellus.

Analisando o gráfico da figura 5 que apresenta os resultados da atividade com o Software Modellus, podemos constatar que a maioria dos alunos responderam as arguições com respostas conexas aos conceitos de Movimento harmônico Simples e de Funções Trigonométricas. Esse fato pode ter sido evidenciado, pois houve o emprego de um Software que integra os conceitos de Funções Trigonométricas com os de Movimento Harmônico Simples.

A atividade desenvolvida no Laboratório de Informática Educativa - LIED consistia na aplicação de uma animação do movimento harmônico simples. Essa atividade possuía três questionamentos dissertativos, partindo da seguinte orientação: "No sentido de iniciar a atividade, abrir o arquivo 'mhs_pêndulo'. Após aberto, o aluno poderá visualizar, na tela da atividade, os gráficos e a animação das funções: $\mathrm{x}=\mathrm{L} \sin$ (teta) e $\mathrm{y}=-\mathrm{L} \cos$ (teta)", conforme a Figura 6.

O primeiro questionamento da atividade solicitava que os alunos realizassem uma descrição do movimento do pêndulo. As respostas dos alunos foram as mais diversificadas, porém oito desses alunos, além de descreverem corretamente como se comportava o movimento harmônico simples, fizeram um comparativo e análises das funções trigonométricas que caracterizam o movimento do pêndulo, como podemos verificar na Figura 7:

Analisando as transcrições dos áudios e a resposta de um aluno, observamos que o mesmo destaca que o movimento do pêndulo se caracteriza pelas funções seno e cosseno e que as duas funções possuem amplitudes distintas. Além disso, vale ressaltar que os alunos descreveram que era possível observar no movimento do pêndulo, em informações matemáticas, se tratava de uma função trigonométrica e que a imagem da função era um intervalo entre $[-5,5]$; em informações físicas, os alunos destacaram que se tratava de um movimento harmônico simples e que existia uma periodicidade.

De acordo com os autores [16], o movimento harmônico simples é projeção do movimento circular uniforme no diâmetro da circunferência na qual acontece o movimento circular. Nesse sentido, o gráfico das funções trigonométricas seno e cosseno, apresentadas no pêndulo, é a projeção, no eixo cartesiano, do movimento circular uniforme da posição em função do tempo.

A atividade solicitava que os alunos comparassem os gráficos das funções seno e cosseno, evidenciando as semelhanças e distinções, como podemos verificar por meio da Figura 8:

Analisando as transcrições dos áudios, do aluno que ofereceu a resposta da figura 8, o mesmo expressou que a amplitude desenvolvida pela função seno na animação era o dobro da função cosseno. Nessa atividade, cinco alunos apresentaram respostas dentro das concepções conceituais de função trigonométricas e de movimento harmônico simples, apesar dos erros de grafias. No entanto, me chamou a atenção à ausência de respostas de dois alunos que deixaram esse item em branco. Estudando as transcrições das conversas durante a pesquisa, percebemos que os dois alunos não responderam a questão porque tinham percebido apenas semelhanças e não diferenças.

Os demais alunos escreveram que a semelhança estava no fato de que as duas formavam um gráfico em ondas e a diferença era que uma possuía amplitude maior que a outra. Ao desenvolver o questionário prévio os alunos não conseguiram descrever semelhanças entre movimentos periódicos, tampouco conseguiram relacionar amplitudes diferentes, pois parte dos alunos não tinha o conceito de amplitude estruturado. Após trabalhar no Modellus, pode-se notar que a representação gráfica em tempo real das variáveis associadas ao movimento periódico contribuiu para que os alunos pudessem identificar semelhanças entre funções.

Essa percepção foi possível, pois "o Modellus tem como função primeira organizar as ideias gerais em torno de conceitos mais específicos, e apresentá-los com uma roupagem menos 'abstrata' [...]" [18]. Para [19] a função seno é ímpar e cosseno é uma função par de período $2 \pi$ e se $\mathrm{f}$ $(\mathrm{x})=\sin (\mathrm{x})$, então $f\left(x+\frac{\pi}{2}\right)=\cos (\mathrm{x}), \log \mathrm{o}$, o gráfico da função cosseno é uma translação de $\frac{\pi}{2}$ do gráfico da função seno. Relacionando com o movimento harmônico simples, x representa a frequência angular do movimento e $\frac{\pi}{2}$ a constante de fase [20].

A terceira pergunta da atividade buscava que o aluno relacionasse o número de períodos que as funções seno e cosseno desenvolveram no final da simulação. Verifiquei que todos os alunos conseguiram identificar o número de períodos desenvolvidos pelas funções trigonométricas. Houve respostas como o da Figura 9 em que o aluno 


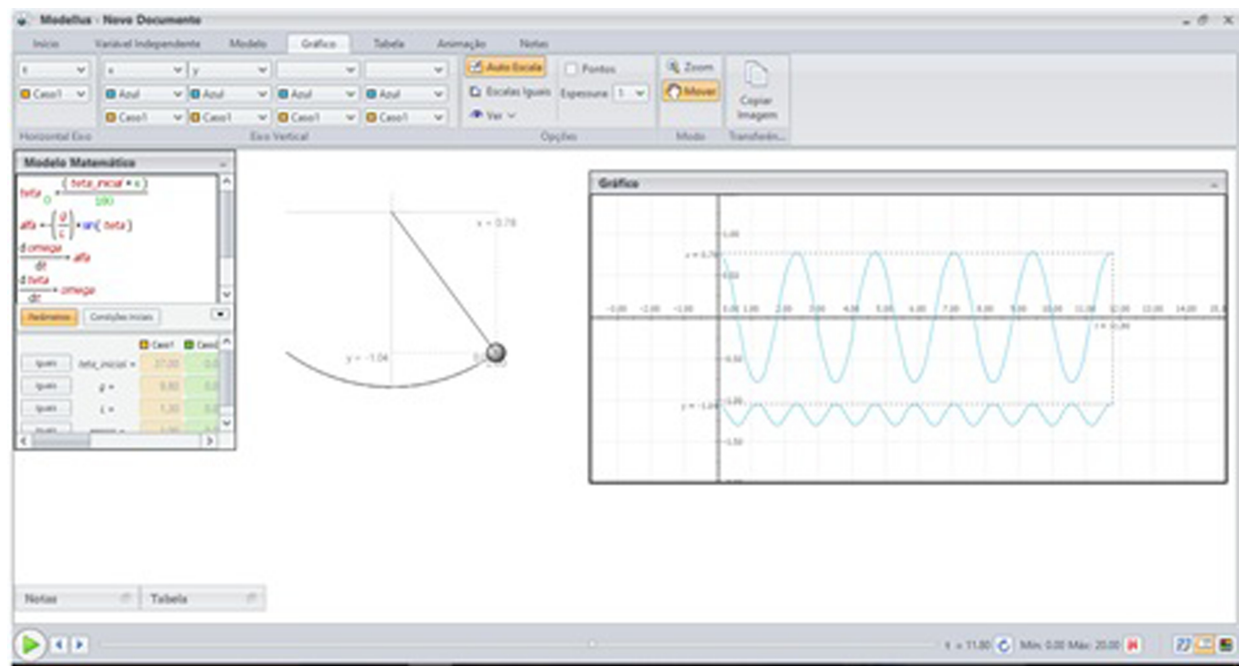

Figura 6: Vista no Modellus da atividade com o Software Modellus, dos autores com a utilização do Software Modellus (2017).

a) Descreva as caracteristicas do movimento do pêndulo.

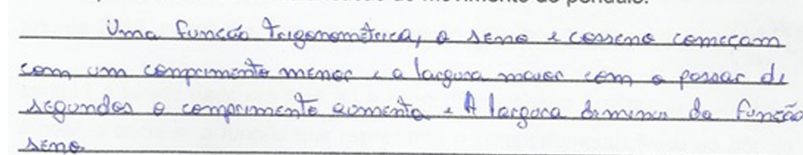

Figura 7: Resposta de um aluno que apresentou convergência, dos autores (2017).

b) Quanto ao gráfico das funçōes seno e cosseno do pêndulo quais os pontos de semelhança e distinção podemos perceber?

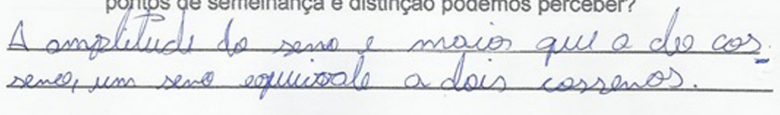

Figura 8: Resposta de um aluno que apresentou convergência, dos autores (2017).

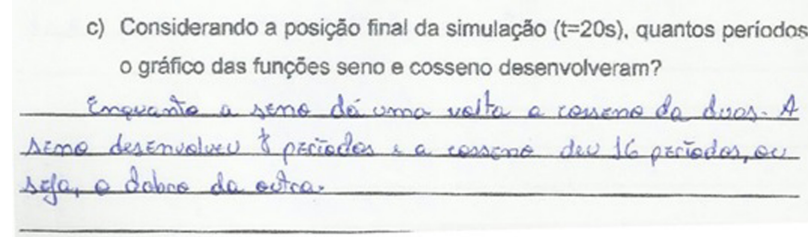

Figura 9: Resposta de um aluno que apresentou convergência, dos autores (2017).

relacionou o comportamento gráfico das funções seno e cosseno.

Segundo [20]:

[...] o tempo que leva para um objeto deslocado executar um ciclo completo de movimento oscilatório de um extremo ao outro e de volta ao anterior é chamado de período T. O inverso do período é a frequência f, que é o número de ciclos por unidade tempo [...].
Além disso, o comportamento oscilatório do pêndulo simples da atividade exploratória é determinado pelas funções seno e cosseno. Analisando os gráficos, é possível verificar que a função seno possui uma amplitude maior que a função cosseno; consequentemente a isso, o ciclo de oscilação da função seno é o dobro da função cosseno. Percebemos, pelas respostas dos alunos, que houve compreensão quanto ao número de períodos desenvolvidos pelas funções seno e cosseno. [11] ressalta que a combinação da representação do real com a forma de apresentação do gráfico da função auxilia o estudante a compreender os conceitos de Matemática e Física que são muitas vezes ensinados por meios de regras descontextualizadas e sem aplicações práticas.

\section{Considerações Finais}

Entre as diferentes formas de emprego dos recursos tecnológicos no ensino de Matemática e Física, apresentadas na literatura, escolhemos a aplicação de um software que possibilitasse a integração dos conceitos de funções trigonométricas com o movimento harmônico simples. Em meio aos diversos tipos de software empregados no ensino de Matemática e Física, optamos pelo uso do Modellus, pois é um software de distribuição gratuita, que não exige do aluno um conhecimento de linguagem de programação. Esse software possibilitou aos alunos uma interação dos conceitos funções trigonométricas e movimento harmônico simples, durante o processo de exploração dos modelos matemáticos.

Ao comparamos os resultados da atividade do questionário estruturado prévio com o da atividade com o Software Modellus, constatamos que os alunos puderam desenvolver atividades diferenciadas, ou seja, esse Software possibilitou uma nova abordagem do conteúdo. Propiciou desenvolver, em conjunto, a função trigonométrica e o movimento harmônico simples. De acordo com [12], no processo de ensino e de aprendizagem de Matemática 
e Física, o auxílio que os recursos tecnológicos podem trazer, por intermédio de softwares educativos está no fato de proporcionarem maior compreensão e facilidade no desenvolvimento de atividades mais complexas.

Nessa perspectiva, o uso do Software Modellus como recurso para compreensão de funções trigonométricas integradas ao movimento harmônico simples revelou-se uma ferramenta de auxílio na compreensão e aplicação desses conceitos. Ao invés de disseminar a Matemática e a Física como ciências prontas e acabadas, podemos conceber um ambiente de aprendizagem informatizado, em que os alunos possam desafiar a criatividade, construir e experimentar hipóteses [12].

As aplicações do questionário estruturado prévio e do questionário de avaliação proporcionaram coletar dados qualitativos. Esses dados corroboraram para que, por meio das atividades realizadas com o uso do Software Modellus, alguns conceitos de funções trigonométricas e de movimento harmônico passassem a ter sentido e aplicabilidade para os alunos participantes da pesquisa.

Em diversos momentos, durante as atividades exploratórias, os alunos declararam que "a matemática ficava mais fácil e significativa"; "os significados dos cálculos eram mais próximo da realidade"; "usar o software nas aulas de matemática facilita o entendimento"; "agora eu consigo entender Matemática e Física". Cabe ressaltar, também, que nem todos os alunos gostaram das atividades.

Finda a investigação, podemos afirmar que a aplicação do software Modellus contribui com a interação do ensino de Matemática com o de Física, podendo promover uma possibilidade diferenciada para a compreensão de diversos conteúdos dessas duas áreas do conhecimento, mesmo tendo uma evolução lenta como foi observado na intervenção, porém com sentido positivo. Assim, a opção de utilizar software educativo facilitou a implantação dessa proposta, pois, muitas instituições de ensino disponibilizam laboratório de informática educativa, que pode ser utilizado para agregar a conexão existente entre o ensino de funções trigonométricas com o de movimento harmônico simples.

\section{Referências}

[1] M.C. Borba, R.S.R. Silva e G. Gadanidis, Fases das Tecnologias Digitais em Educação Matemática: Sala de Aula e Internet em Movimento (Autêntica, Belo Horizonte, 2014).

[2] M.T. Quartieri, M.M. Dullius e I.M. Giongo, Rev. Educação Matemática em Revista, 1, 25 (2012).

[3] M.A.A. Pacheco e W.L.A. Barbosa, in: XX Simpósio Nacional do Ensino de Fúsica, São Paulo, 2013 (Sociedade Brasileira de Física, São Paulo, 2013) p. 495.

[4] M.M. Soffa e P.R.C. Alcântara, O Uso do Software Educativo: Reflexões da Prática Docente na Sala Informatizada (PUCPR, Curitiba, 2008).

[5] I.F. Druck, M.C. Bonomi, V. Giampaoli, A.P. Jahn e I.M. Dutra, Formação de professores do Ensino Médio,
Etapa II - Caderno V: Matemática (UFPR, Curitiba, 2014), p. 49.

[6] A.D. Trindade, A. Pinto Junior, C.S. Kryszczun, E.S.O. Barra, M.R. Machado, M.A. Gonçalves e M.F.R. Neu, Formação de professores do Ensino Médio, Etapa II Caderno III: Matemática (UFPR, Curitiba, 2014), p. 53.

[7] R.P. Sousa, F.M. Moita e A.B.G. Carvalho, Tecnologias Digitais na Educação (EDUEPB, Campina Grande, 2011).

[8] A.M. Pinto, Rev. Portal Anpedsul. 5, 1 (2004).

[9] I.S. Araujo, E.A. Veit e M.A. Moreira, Rev. Brasileira de Ensino de Física 26, 179 (2004).

[10] J.F. Mendes, O Uso do Software Modellus na Integração Entre Conhecimentos Teóricos e Atividades Experimentais de Tópicos de Mecânica sob a Perspectiva da Aprendizagem Significativa. Dissertação de Mestrado Profissional em Ensino de Ciências, UNB, 2009.

[11] D.C.M. Torresan, O Uso do Software de Simulação Modellus na Conceitualização de Derivada: Experiências de Ensino-Aprendizagem com base em Vergnaud. Dissertação de Mestrado em Ensino de Ciências e Matemática, Universidade Luterana do Brasil, 2008.

[12] P.A.F. Novais e L.F. Simião, in: IX Encontro Regional de Educação Matemática, Ijuí, 2011 (Editora Unijuí, Ijuí, 2011), 1.

[13] L. Chwif e A.C. Medina, Modelagem e Simulação de Eventos Discretos: Teoria e Aplicações.(Editora do Autor, São Paulo, 2010)

[14] M.C. Borba e J.L. Araújo, Pesquisa Qualitativa em Educação Matemática (Autêntica, Belo Horizonte, 2013).

[15] H.L. Guidorizzi, Um Curso de Cálculo (LTC, Rio de Janeiro, 2011), v. 1, $5^{\text {a }}$ ed.

[16] D. Halliday, R. Resnick e J. Walker, Fundamentos de Física, Vol. 1 (LTC, Rio de Janeiro, 2012).

[17] G. Iezzi, Fundamentos da Matemática Elementar (Atual, São Paulo, 2013), v. 3, $9^{\text {a }}$ ed.

[18] J.R.G. Costa Junior, A.C. Silva e A.V.S. Brito, in: $X X$ Simpósio Nacional do Ensino de Física, São Paulo, 2013 (Sociedade Brasileira de Física, São Paulo, 2013), p. 1003.

[19] G. Ávila, Cálculo das Funções de Uma Variável (LTC, Rio de Janeiro, 2003), vol. $1,7^{\mathrm{a}}$ ed.

[20] P.A. Tipler e G. Mosca, Física para Cientistas e Engenheiros: Mecânica Oscilações e Ondas Termodinâmicas, Vol. 1 (LTC, Rio de Janeiro, 2010), $6^{\text {a }}$ ed. 